\title{
Signal Models for TDOA/FDOA Estimation
}

\author{
Mark L. Fowler and Xi Hu \\ Department of Electrical and Computer Engineering \\ State University of New York at Binghamton \\ Binghamton, NY
}

\begin{abstract}
Much research has been done in the area of estimating time-difference-of-arrival (TDOA) and frequency-difference-of-arrival (FDOA) and their use in locating a radiating source. Early work in this area was focused on locating acoustic sources using passive sonar processing. Only later was TDOA/FDOA-based location considered for the case of passively locating electromagnetic sources. As a result of this, it is tempting to use results derived for the acoustic case when answering questions about the electromagnetic case. This correspondence shows that such borrowing can lead to incorrect results. The key factor that drives the significant differences between these two cases is the difference between the signal model assumptions for the two cases: WSS Gaussian process in the acoustic case and a deterministic signal in the electromagnetic case. Although the received signal equations may look identical (showing delay and Doppler shift), the resulting Fisher information, Cramer-Rao bound, and maximum likelihood estimator (MLE) are fundamentally different for the two signal scenarios.
\end{abstract}

Index Terms: TDOA/FDOA Estimation, Emitter Location, Fisher Information, Cramer-Rao Bound, ML Estimation

Corresponding Author:

Mark Fowler

Department of Electrical and Computer Engineering

State University of New York at Binghamton

P. O. Box 6000

Binghamton, NY 13902-6000

Phone: 607-777-6973

Fax: 607-777-4464

E-mail: mfowler@binghamton.edu 


\section{Introduction}

The location of a source can be determined from signals intercepted at several sensors. One of the most effective methods is to use estimates of the time-difference-of-arrival (TDOA) and/or the frequencydifference-of-arrival (FDOA) between pairs of signals received at the sensors. This involves a sequence of two estimation problems: (i) processing the signals to give a set of TDOA/FDOA estimates (e.g., [1], [3], [4], [5], [6], [7], [8], [10], [12], [13], [14] ), and (ii) processing the resulting TDOA/FDOA estimates to estimate the location (e.g., [2], [9], [11]).

Optimal processing for the second stage requires an understanding of the probabilistic characteristics of the TDOA/FDOA estimates from the first stage. Therefore, much work has been done to derive optimal TDOA/FDOA estimates and to characterize their covariance matrix. However, as this correspondence will point out, when using results from the many papers on TDOA/FDOA estimation it is important to understand the differences that arise due to the different signal models that have been used. TDOA/FDOA results were first developed in the early 1970s for the case of passively locating underwater acoustic sources, where the accepted model for the signal is a WSS random process (almost always assumed Gaussian) [1], [3], [4], [5], [6], [8], [10], [12]. Only later was TDOA/FDOAbased location considered for the case of passively locating electromagnetic sources [7], [9], [11], [14]. For electromagnetic sources such as radar and communication transmitters, a stationary random process is generally deemed inappropriate and a deterministic signal model may be better [14]. Stein [14] considers the development of the ML estimator for the deterministic case (but did not develop the Cramer-Rao bound for that case) and Quazi [6] briefly addresses the deterministic vs. random signal differences but only in the context between active vs. passive location systems (he does not mention differences between passive systems for the acoustic and electromagnetic scenarios). In general, there 
seems to be confusion and unawareness about the differences between the passive acoustic case and the passive electromagnetic case ${ }^{1}$. For example, many times acoustic-signal results have been misused in the electromagnetic scenario; this seems to occur more often than the reverse -likely due to the fact that the acoustic setting was the first explored and has generated many widely-known publications.

Section II discusses the differences between the signal models and explores the impact these differences have on the underlying PDF, the Fisher information matrix (FIM), the Cramer-Rao Bound (CRB), and the maximum likelihood estimator (MLE). The Appendix derives specific results for evaluating the FIM for the electromagnetic scenario.

\section{Impact of Signal Model on TDOA/FDOA Estimation Results}

\section{A. Signal Models}

The model for two sampled passively-received complex baseband signals at two sensors is given by

$$
\begin{aligned}
& r_{1}[n]=s\left(n T-\tau_{1}\right) e^{j \nu_{n} n T}+w_{1}[n] \\
& r_{2}[n]=s\left(n T-\tau_{2}\right) e^{j \nu_{2} n T}+w_{2}[n]
\end{aligned}
$$

where $s(t)$ is the complex envelope of the continuous-time transmitted signal, $T$ is the sampling interval, the $w_{i}[n]$ are corrupting noises, $\tau_{1}$ and $\tau_{2}$ are delays, and $v_{1}$ and $v_{2}$ are Doppler shifts. It should be mentioned that for electromagnetic signals it is usually appropriate to use Doppler shift to model the effect of motion between source and receiver, but for acoustic signals it is often not appropriate to use Doppler shift; however, to allow easier focus on the statistical model differences we assume here that this is valid. The TDOA $\Delta_{\tau}=\tau_{1}-\tau_{2}$ and the FDOA $\Delta_{v}=v_{1}-v_{2}$ are the parameters to be estimated from time-domain samples of these signals; we define $\boldsymbol{\theta}=\left[\begin{array}{ll}\Delta_{\tau} & \Delta_{v}\end{array}\right]^{T}$. For both the acoustic scenario and the electromagnetic scenario, the accepted modeling as-

\footnotetext{
${ }^{1}$ We use the terms "acoustic" and "electromagnetic" as merely convenient labels that arise from the historical development of TDOA/FDOA results; it is of course possible to have an acoustic signal that is better modeled as deterministic or
} 
sumptions for the noises $w_{i}[n]$ are (i) they are zero-mean WSS random processes, (ii) they are each Gaussian, and (iii) they are independent of each other. In general they are not necessarily assumed to be white, but that is a common assumption. For notational purposes: (i) the signal $s_{i}[n]=s\left(n T-\tau_{i}\right) e^{j v_{i} n T}$, (ii) the vector $\mathbf{r}_{i}$ is the vector with elements that are the values of $r_{i}[n]$, and (iii) the vector $\mathbf{r}$ is $\mathbf{r}=\left[\begin{array}{ll}\mathbf{r}_{1}^{T} & \mathbf{r}_{2}^{T}\end{array}\right]^{T}$; the vector $\mathbf{s}$ is defined as $\mathbf{s}_{\boldsymbol{\theta}}=\left[\begin{array}{ll}\mathbf{s}_{1}^{T} & \mathbf{s}_{2}^{T}\end{array}\right]^{T}$, where we explicitly notate the dependence on the TDOA/FDOA parameter vector $\boldsymbol{\theta}$.

This much is common between the acoustic and electromagnetic scenarios. The differences arise in what is assumed about the signal $s_{i}[n]$. For the acoustic scenario the accepted modeling assumptions on the signal $s_{i}[n]$ are: (i) it is a zero-mean WSS random process, (ii) it is Gaussian, (iii) it is independent of each noise process, and (iv) it need not be assumed white, although that is a special case that is often considered. This randomsignal model arose due to the fact that the early TDOA/FDOA researchers were investigating passive sonar, where the acoustic signals were made by the motors of ocean vehicles. For this scenario: (i) the WSS random process assumption is consistent with the erratic nature of the motor sounds, (ii) the Gaussian assumption is motivated by (perhaps) the central limit theorem and (certainly) the tractability it provides, and (iii) the independence of signal and the noises is reasonable based on physical considerations.

There are very few published fundamental results on TDOA/FDOA estimation for electromagnetic signals (e.g., [7], [14]). Signals emitted by electromagnetic sources tend to have much more regular structure than the erratic variations seen in acoustic signals made by ocean vehicles; therefore they don't readily evoke the notion of random process. Still, a classic example of a WSS random process is a sinusoidal signal with uniformly distributed phase; despite the fact that each realization of this process exhibits very regular structure it is a WSS random process. Similarly, radar pulse trains can be viewed as random processes for the very same reason: they can be modeled as having random transmission parameters (e.g., random time offset, random phase offset, etc.). However, such signals - with their widely spaced pulses - can hardly be thought to be WSS processes (e.g., variance within a pulse is not equal to the variance between pulses). Furthermore, they certainly cannot 
be modeled as Gaussian, and finding some other suitable probability model seems daunting and is generally fruitless when one tries to solve problems using such a probability model. Similar arguments could be made for many other types of electromagnetic transmitted signals, though not for all. Thus, in such a scenario it is prudent to consider the signal $s_{i}[n]$ to be a deterministic signal rather than a random signal [14].

When we consider the estimates of TDOA and FDOA, $\hat{\Delta}_{\tau}$ and $\hat{\Delta}_{v}$, we typically wish to find unbiased estimates that minimize $E\left\{\left(\hat{\Delta}_{\tau}-\Delta_{\tau}\right)^{2}\right\}$ and $E\left\{\left(\hat{\Delta}_{v}-\Delta_{v}\right)^{2}\right\}$. Here is an immediate fundamental distinction between these two models: it needs to be understood that when the signal is random these expectations are taken over the combined ensemble of signal and noise whereas when the signal is deterministic these expectations are taken over only the noise ensemble. Thus, when the signal is random we are finding the average squared error over all possible noises and signals (within the ensemble); when the signal is deterministic we are finding the average squared error over all possible noises for one specific signal. Furthermore, in simulations for the random signal case, in each Monte Carlo run the signal is selected from its ensemble and the noise is selected from its ensemble; whereas for the deterministic signal case the same signal is used in every Monte Carlo run.

\section{B. PDFs Under the Signal Models}

Despite these differences, from the above discussion we see that for both models the received data vector $\mathbf{r}$ is Gaussian and has a Gaussian PDF. The key distinction between these two scenarios that drives all the differences in the FIM, the CRB, and the MLE processing is the manner in which the TDOA/FDOA impacts the parameters of the Gaussian PDF of data vector $\mathbf{r}$. For the case of the acoustic scenario, the mean of $\mathbf{r}$ is zero and the covariance matrix of $\mathbf{r}$ depends on TDOA/FDOA, so we denote it as $\mathbf{C}_{\boldsymbol{\theta}}$ to show that dependence. In contrast, for the case of the electromagnetic scenario, the mean of $\mathbf{r}$ is $\mathbf{s}_{\boldsymbol{\theta}}=\left[\begin{array}{ll}\mathbf{s}_{1}^{T} & \mathbf{s}_{2}^{T}\end{array}\right]^{T}$ which depends on TDOA/FDOA and the covariance matrix of $\mathbf{r}$ is a block diagonal matrix of the two individual noise covariance matrices, and therefore does not depend on TDOA/FDOA so we denote it as $\mathbf{C}$ in this case. From this single distinction we see that the PDF for the acoustic case is 


$$
p_{a c}(\mathbf{r} ; \boldsymbol{\theta})=\frac{1}{\operatorname{det}\left(\pi \mathbf{C}_{\boldsymbol{\theta}}\right)} \exp \left\{-\mathbf{r}^{H} \mathbf{C}_{\boldsymbol{\theta}}^{-1} \mathbf{r}\right\}
$$

and the PDF for the electromagnetic case is

$$
p_{e m}(\mathbf{r} ; \boldsymbol{\theta})=\frac{1}{\operatorname{det}(\pi \mathbf{C})} \exp \left\{-\left(\mathbf{r}-\mathbf{s}_{\boldsymbol{\theta}}\right)^{H} \mathbf{C}^{-1}\left(\mathbf{r}-\mathbf{s}_{\boldsymbol{\theta}}\right)\right\}
$$

The differences between the PDFs for these two signal model scenarios is clearly evident in (2) and (3); it is this difference that leads to significant differences in the structures of Cramer-Rao bounds as well as the maximum likelihood estimators for the two cases. It should be observed that these are each a special case of the complex general Gaussian case (see Ch. 15 of [15] for the complex data case covered here) given by

$$
p_{g g}(\mathbf{r} ; \boldsymbol{\theta})=\frac{1}{\operatorname{det}\left(\pi \mathbf{C}_{\boldsymbol{\theta}}\right)} \exp \left\{-\left(\mathbf{r}-\boldsymbol{\mu}_{\boldsymbol{\theta}}\right)^{H} \mathbf{C}_{\boldsymbol{\theta}}^{-1}\left(\mathbf{r}-\boldsymbol{\mu}_{\boldsymbol{\theta}}\right)\right\}
$$

where both the mean $\boldsymbol{\mu}_{\boldsymbol{\theta}}$ and the covariance $\mathbf{C}_{\boldsymbol{\theta}}$ of $\mathbf{r}$ depend on the parameter vector $\boldsymbol{\theta}$.

\section{Fisher Information and Cramer-Rao Bound}

The elements of the Fisher information matrix $\mathbf{J}_{g g}$ for the complex general Gaussian scenario PDF in (4) is a standard result [15] given by

$$
\left[J_{g g}\right]_{i j}=2 \operatorname{Re}\left(\left[\frac{\partial \boldsymbol{\mu}_{\boldsymbol{\theta}}}{\partial \theta_{i}}\right]^{H} \mathbf{C}^{-1}(\boldsymbol{\theta})\left[\frac{\partial \boldsymbol{\mu}_{\boldsymbol{\theta}}}{\partial \theta_{j}}\right]\right)+\operatorname{tr}\left(\mathbf{C}_{\boldsymbol{\theta}}^{-1} \frac{\partial \mathbf{C}_{\boldsymbol{\theta}}}{\partial \theta_{i}} \mathbf{C}_{\boldsymbol{\theta}}^{-1} \frac{\partial \mathbf{C}_{\boldsymbol{\theta}}}{\partial \theta_{j}}\right)
$$

Notice that there are two terms in this result: one that depends on the sensitivity of the mean to the parameters and one that depends on the sensitivity of the covariance to the parameters. Because the acoustic and electromagnetic scenarios are two different special cases of the generalized Gaussian scenario, we can use the result in (5) to find the result for each of these two special cases.

As discussed above, for the acoustic scenario the mean of $\mathbf{r}$ is zero and therefore does not depend on the parameter vector; thus the first term in (5) is zero and the Fisher information matrix $\mathbf{J}_{a c}$ for the acoustic scenario is then given by 


$$
\left[\mathbf{J}_{a c}\right]_{i j}=\operatorname{tr}\left[\mathbf{C}_{\boldsymbol{\theta}}^{-1} \frac{\partial \mathbf{C}_{\boldsymbol{\theta}}}{\partial \theta_{i}} \mathbf{C}_{\boldsymbol{\theta}}^{-1} \frac{\partial \mathbf{C}_{\boldsymbol{\theta}}}{\partial \theta_{j}}\right]
$$

which gives a result that is well known in the TDOA/FDOA literature for the acoustic signal scenario (e.g., [8], [10]). The corresponding CRBs are found by inverting the respective FIM; doing that directly is difficult but equivalent approaches that avoid direct use of (6) have been found (see for example [10]).

For the electromagnetic scenario the covariance of $\mathbf{r}$ doesn't depend on the parameter vector; thus, the second term in (5) is zero and the Fisher information matrix $\mathbf{J}_{e m}$ for the electromagnetic scenario is thus given by

$$
\left[\mathbf{J}_{e m}\right]_{i j}=2 \operatorname{Re}\left\{\frac{\partial \mathbf{s}_{\boldsymbol{\theta}}^{H}}{\partial \theta_{i}} \mathbf{C}^{-1} \frac{\partial \mathbf{s}_{\boldsymbol{\theta}}}{\partial \theta_{j}}\right\}
$$

which surprisingly is not widely seen in the TDOA/FDOA literature for the electromagnetic signal scenario. See the Appendix for details of evaluating this form to compute the FIM for TDOA/FDOA under the deterministic signal case. The corresponding CRBs are found by inverting the respective FIM; doing that using (7) leads to forms that are similar but not identical to those given without proof in [7] for the continuous-time case.

Comparing (6) and (7) we see that there is a significant difference between the structures of the FIM for the two cases. In particular, for the acoustic scenario it is convenient to use "Whittle's Theorem" to write the Fisher information for a WSS process in the spectral domain [12]; however, this alternative approach for the WSS-signal case can not be used for the electromagnetic scenario. An even more important distinction is as follows. As pointed out in several publications (e.g., [8], [10], [12]) the off-diagonal elements of the acoustic scenario FIM in (6) are zero under the mild assumption of a large time-bandwidth product, thus indicating that for the acoustic scenario the optimal estimate of TDOA should be uncorrelated with the optimal estimate of FDOA. However, the electromagnetic scenario FIM in (7) does not, in general, yield this uncorrelated TDOA/FDOA condition. For example, the Appendix shows that for the case of white noise the result in (7) gives the off-diagonal FI element as

$$
\left[\mathbf{J}_{e m}\right]_{12}=2 \operatorname{Re}\left\{\frac{1}{\sigma_{1}^{2}} \sum_{n}-j n T s^{*}\left(n T-\tau_{1}\right) s^{\prime}\left(n T-\tau_{1}\right)+\frac{1}{\sigma_{2}^{2}} \sum_{n}-j n T s^{*}\left(n T-\tau_{2}\right) s^{\prime}\left(n T-\tau_{2}\right)\right\},
$$


where $s^{\prime}(t)$ is the derivative of $s(t)$. From this result we see that the off-diagonal element in general is not zero; as an illustration, the linear chirp signal has been shown to have non-zero cross-FI [16]. Thus, for the acoustic case we can expect that for a pair of sensors the optimal TDOA estimate is uncorrelated from the optimal FDOA estimate but that should not be expected in the electromagnetic case.

An important impact of this comes when assessing the location accuracy that can be achieved from a set of TDOA/FDOA measurements. Assuming an ML estimator for the TDOA/FDOA values, their estimates can be taken to be Gaussian and then the CRB on the location estimate covariance becomes

$$
\mathbf{C}_{l o c}=\left(\mathbf{G}^{T} \mathbf{J G}\right)^{-1}
$$

where $\mathbf{J}$ is the FIM for all $N$ TDOA/FDOA measurements and $\mathbf{G}$ is the Jacobian of the TDOA/FDOA values with respect to the emitter's location coordinates [11], [17]. From (9) it is clear that when performing studies of location accuracy, using the incorrect FIM for TDOA/FDOA - i.e., using the acoustic FIM when the electromagnetic FIM should be used, or vice versa - can lead to incorrect conclusions about location accuracy.

\section{Maximum Likelihood Estimator}

The ML estimator is found by maximizing the log likelihood function (LLF), which is typically expressed by taking partial derivatives of the LLF with respect to each parameter and setting them equal to zero. For the complex generalized Gaussian case there is a standard result (see Sect. 15.7 of [15]) given by

$$
\frac{\partial \ln \left\{p_{g g}(\mathbf{r} ; \boldsymbol{\theta})\right\}}{\partial \theta_{i}}=-\operatorname{tr}\left(\mathbf{C}_{\boldsymbol{\theta}}^{-1} \frac{\partial \mathbf{C}_{\boldsymbol{\theta}}}{\partial \theta_{i}}\right)+2 \operatorname{Re}\left\{\left[\mathbf{r}-\boldsymbol{\mu}_{\boldsymbol{\theta}}\right]^{H} \mathbf{C}_{\boldsymbol{\theta}}^{-1} \frac{\partial \boldsymbol{\mu}_{\boldsymbol{\theta}}}{\partial \theta_{i}}\right\}+\left[\mathbf{r}-\boldsymbol{\mu}_{\boldsymbol{\theta}}\right]^{H} \mathbf{C}_{\boldsymbol{\theta}}^{-1} \frac{\partial \mathbf{C}_{\boldsymbol{\theta}}}{\partial \theta_{i}} \mathbf{C}_{\boldsymbol{\theta}}^{-1}\left[\mathbf{r}-\boldsymbol{\mu}_{\boldsymbol{\theta}}\right]
$$

Notice that there are three terms in this result: one that depends on the sensitivity of the mean to the parameters and two that depend on the sensitivity of the covariance to the parameters. Because the acoustic and electromagnetic scenarios are two different special cases of the generalized Gaussian scenario, we can use the result in (10) to find the result for each of these two special cases.

For the acoustic scenario the mean of $\mathbf{r}$ is zero and therefore does not depend on the parameter vector; thus, the partial derivatives of the LLF for (2) are given by just two terms from (10): 


$$
\begin{aligned}
\frac{\partial p_{a c}(\mathbf{r} ; \boldsymbol{\theta})}{\partial \theta_{i}} & =-\frac{\partial}{\partial \theta_{i}} \ln \left[\operatorname{det}\left(\pi \mathbf{C}_{\boldsymbol{\theta}}\right)\right]-\frac{\partial}{\partial \theta_{i}} \mathbf{r}^{H} \mathbf{C}_{\boldsymbol{\theta}}^{-1} \mathbf{r} \\
& =-\operatorname{tr}\left(\mathbf{C}_{\boldsymbol{\theta}}^{-1} \frac{\partial \mathbf{C}_{\boldsymbol{\theta}}}{\partial \theta_{i}}\right)+\mathbf{r}^{H} \mathbf{C}_{\boldsymbol{\theta}}^{-1} \frac{\partial \mathbf{C}_{\boldsymbol{\theta}}}{\partial \theta_{i}} \mathbf{C}_{\boldsymbol{\theta}}^{-1} \mathbf{r}
\end{aligned}
$$

This result is well known in the passive sonar literature (e.g., see [8],[10]). In [10] it is further stated that for the TDOA/FDOA case the determinant term in the first line of (11) does not depend on the parameter vector; therefore, the first term in the second line of (11) can be ignored to give

$$
\frac{\partial p_{a c}(\mathbf{r} ; \boldsymbol{\theta})}{\partial \theta_{i}}=\mathbf{r}^{H} \mathbf{C}_{\boldsymbol{\theta}}^{-1} \frac{\partial \mathbf{C}_{\boldsymbol{\theta}}}{\partial \theta_{i}} \mathbf{C}_{\boldsymbol{\theta}}^{-1} \mathbf{r} .
$$

It should be noted that finding the value of $\theta$ that drives (12) to zero is equivalent to

$$
\hat{\boldsymbol{\theta}}_{M L, a c}=\underset{\boldsymbol{\theta}}{\arg \max }\left\{-\mathbf{r}^{H} \mathbf{C}_{\boldsymbol{\theta}}^{-1} \mathbf{r}\right\}
$$

For the electromagnetic scenario the covariance of $\mathbf{r}$ does not depend on the parameter vector; thus, the partial derivatives of the LLF for (3) are given by the second term in (10)

$$
\frac{\partial p_{e m}(\mathbf{r} ; \boldsymbol{\theta})}{\partial \theta_{i}}=2 \operatorname{Re}\left\{\left[\mathbf{r}-\mathbf{s}_{\boldsymbol{\theta}}\right]^{H} \mathbf{C}^{-1} \frac{\partial \mathbf{s}_{\boldsymbol{\theta}}}{\partial \theta_{i}}\right\} .
$$

It should be noted that finding the value of $\theta$ that drives (14) to zero is equivalent to

$$
\begin{aligned}
\hat{\boldsymbol{\theta}}_{M L, e m} & =\underset{\boldsymbol{\theta}}{\arg \max }\left\{-\left[\mathbf{r}-\mathbf{s}_{\boldsymbol{\theta}}\right]^{H} \mathbf{C}^{-1}\left[\mathbf{r}-\mathbf{s}_{\boldsymbol{\theta}}\right]\right\} \\
& =\underset{\boldsymbol{\theta}}{\arg \max }\left\{2 \operatorname{Re}\left\{\mathbf{r}^{H} \mathbf{C}^{-1} \mathbf{s}_{\boldsymbol{\theta}}\right\}-\mathbf{s}_{\boldsymbol{\theta}}^{H} \mathbf{C}^{-1} \mathbf{s}_{\boldsymbol{\theta}}\right\}
\end{aligned}
$$

Comparing (12) and (13) to (14) and (15) shows that we should expect fundamental differences between the MLE for the acoustic and electromagnetic cases. Surprisingly though, each case results in a structure that involves pre-filtering the received signals followed by cross-correlation (see [3], [10] for the acoustic case and [14] for the electromagnetic case). However, although both cases share this generalized correlator structure, the pre-filtering needed for each case is quite different. For the acoustic case the filters depend on an interplay between the signal PSD and the noise PSD [3], [10], whereas for the electromagnetic case the filters depend only on the noise PSD and not on the signal's spectral structure [14]. As a result, the acoustic generalized correlator 
simplifies to a standard correlator only when noise and signal are white [3], [5] whereas the electromagnetic generalized correlator simplifies to a standard correlator whenever the noise is white, regardless of the signal's spectral structure [14]. Despite this difference in ML structure for the two scenarios there are many cases in the literature that address the electromagnetic case but reference (and sometimes use) the acoustic scenario's generalized correlator when it is not appropriate.

Another interesting difference between the two scenarios is pointed out by Stein [14]. For the deterministic signal, the MLE involves estimating the unknown deterministic signal. It is clear from (15) that this step is necessary since the maximization in (15) involves the unknown signal vector $\mathbf{s}_{\theta}$. The development of the MLE for this scenario naturally includes the estimation of the underlying signal as an intrinsic part of the processing.

Weinstein [8] explored the losses that arise when the ML estimator for TDOA/FDOA is done on a decentralized pairwise basis rather than using all received signals in a centralized processor to jointly estimate all TDOA/FDOA values. Although Weinstein does not explicitly state that he considers only the passive acoustic scenario nor does he state that the signal is modeled as a WSS Gaussian process, the PDF that he uses is given by (2) and not by (3) and therefore all the results in [8] are applicable to the acoustic scenario but not to the electromagnetic scenario. It is not clear that Weinstein's general insights for the near equivalence of decentralized and centralized processing when the SNR is high carries over to the electromagnetic case; the decentralized vs. centralized analysis for the electromagnetic case is more complicated than for the acoustic case and does not seem to lead to easily generalized results.

\section{Conclusions}

The signal models for these two cases (passive acoustic and passive electromagnetic) may seem to be the same at a casual look (i.e., the equations in (1) are used in each case) but the underlying assumption about the signal (i.e., WSS Gaussian signal for the passive acoustic case and a deterministic signal for the passive electromagnetic case) leads to important differences in the results for the FIM, CRB, and MLE. The main differences are that: (i) the general structures of the FIM and CRB are significantly different; (ii) a key specific difference in the FIM/CRB structure is that unlike in the acoustic case, for the electromagnetic case the FDOA and 
TDOA estimates of a signal pair are likely to be correlated; (iii) for the electromagnetic case the MLE is an unfiltered cross-correlator whenever the noise is white (the acoustic case requires the signal to be white in order to remove the filters). Ignoring these differences can lead to incorrect location accuracy assessments as well as improper choices when developing processing schemes.

\section{Appendix: Derivation of FIM for Deterministic Signal Case}

To evaluate the FIM in (7) for the deterministic-signal TDOA/FDOA case we need partial derivatives of the signal vector $\mathbf{s}_{\boldsymbol{\theta}}=\left[\begin{array}{ll}\mathbf{s}_{1}^{T} & \mathbf{s}_{2}^{T}\end{array}\right]^{T}$. Using the signal model in (1) we get

$$
\frac{\partial \mathbf{s}_{\boldsymbol{\theta}}}{\partial \Delta_{\tau}}=\left[\begin{array}{c}
\frac{\partial \mathbf{s}_{1}}{\partial \Delta_{\tau}} \\
\frac{\partial \mathbf{s}_{2}}{\partial \Delta_{\tau}}
\end{array}\right]=\left[\begin{array}{l}
\left(\frac{\partial \mathbf{s}_{1}}{\partial \tau_{1}}\right) /\left(\frac{\partial \Delta_{\tau}}{\partial \tau_{1}}\right) \\
\left(\frac{\partial \mathbf{s}_{2}}{\partial \tau_{2}}\right) /\left(\frac{\partial \Delta_{\tau}}{\partial \tau_{2}}\right)
\end{array}\right]=\left[\begin{array}{c}
\frac{\partial \mathbf{s}_{1}}{\partial \tau_{1}} \\
-\frac{\partial \mathbf{s}_{2}}{\partial \tau_{2}}
\end{array}\right]=\left[\begin{array}{c}
-\mathbf{s}_{1}^{\prime} \\
\mathbf{s}_{2}^{\prime}
\end{array}\right]
$$

where $\mathbf{s}_{i}^{\prime}$ is the vector of samples of the time-derivative of the received signal; we have used the chain rule here and the fact that $\partial s\left(n T-\tau_{i}\right) / \partial \tau_{i}=-d s(t) /\left.d t\right|_{t=n T-\tau_{i}}$. Similarly we have

$$
\frac{\partial \mathbf{s}_{\boldsymbol{\theta}}}{\partial \Delta_{v}}=\left[\begin{array}{c}
\frac{\partial \mathbf{s}_{1}}{\partial \Delta_{v}} \\
\frac{\partial \mathbf{s}_{2}}{\partial \Delta_{v}}
\end{array}\right]=\left[\begin{array}{c}
\frac{\partial \mathbf{s}_{1}}{\partial v_{1}} \\
-\frac{\partial \mathbf{s}_{2}}{\partial v_{2}}
\end{array}\right]=\left[\begin{array}{c}
\tilde{\mathbf{s}}_{1} \\
-\tilde{\mathbf{s}}_{2}
\end{array}\right]
$$

where $\tilde{\mathbf{s}}_{i}$ is the vector with elements that are the elements of $\mathbf{s}_{i}$ multiplied by the corresponding $j n T$ with $n$ running sequentially over its range. Using these results in (7) gives

$$
\begin{gathered}
{\left[\mathbf{J}_{e m}\right]_{11}=2 \operatorname{Re}\left\{\left[\begin{array}{c}
-\mathbf{s}_{1}^{\prime} \\
\mathbf{s}_{2}^{\prime}
\end{array}\right]^{H} \mathbf{C}^{-1}\left[\begin{array}{c}
-\mathbf{s}_{1}^{\prime} \\
\mathbf{s}_{2}^{\prime}
\end{array}\right]\right\}=2 \operatorname{Re}\left\{\mathbf{s}_{1}^{\prime H} \mathbf{C}_{1}^{-1} \mathbf{s}_{1}^{\prime}+\mathbf{s}_{2}^{\prime H} \mathbf{C}_{2}^{-1} \mathbf{s}_{2}^{\prime}\right\},} \\
{\left[\mathbf{J}_{e m}\right]_{22}=2 \operatorname{Re}\left\{\left[\begin{array}{c}
\tilde{\mathbf{s}}_{1} \\
-\tilde{\mathbf{s}}_{2}
\end{array}\right]^{H} \mathbf{C}^{-1}\left[\begin{array}{c}
\tilde{\mathbf{s}}_{1} \\
-\tilde{\mathbf{s}}_{2}
\end{array}\right]\right\}=2 \operatorname{Re}\left\{\tilde{\mathbf{s}}_{1}^{H} \mathbf{C}_{1}^{-1} \tilde{\mathbf{s}}_{1}+\tilde{\mathbf{s}}_{2}^{H} \mathbf{C}_{2}^{-1} \tilde{\mathbf{s}}_{2}\right\},}
\end{gathered}
$$




$$
\left[\mathbf{J}_{e m}\right]_{12}=\left[\mathbf{J}_{e m}\right]_{21}=2 \operatorname{Re}\left\{\left[\begin{array}{c}
-\mathbf{s}_{1}^{\prime} \\
\mathbf{s}_{2}^{\prime}
\end{array}\right]^{H} \mathbf{C}^{-1}\left[\begin{array}{c}
\tilde{\mathbf{s}}_{1} \\
-\tilde{\mathbf{s}}_{2}
\end{array}\right]\right\}=2 \operatorname{Re}\left\{-\mathbf{s}_{1}^{\prime H} \mathbf{C}_{1}^{-1} \tilde{\mathbf{s}}_{1}-\mathbf{s}_{2}^{\prime H} \mathbf{C}_{2}^{-1} \tilde{\mathbf{s}}_{2}\right\},
$$

where $\mathbf{C}_{i}$ is the covariance of the $i^{\text {th }}$ noise process. For white noise with noise variances of $\sigma_{i}^{2}$ we get

$$
\begin{gathered}
{\left[\mathbf{J}_{e m}\right]_{11}=\frac{2}{\sigma_{1}^{2}} \sum_{n}\left|s^{\prime}\left(n T-\tau_{1}\right)\right|^{2}+\frac{2}{\sigma_{2}^{2}} \sum_{n}\left|s^{\prime}\left(n T-\tau_{2}\right)\right|^{2},} \\
{\left[\mathbf{J}_{e m}\right]_{22}=\frac{2 T}{\sigma_{1}^{2}} \sum_{n} n^{2}\left|s\left(n T-\tau_{1}\right)\right|^{2}+\frac{2}{\sigma_{2}^{2}} \sum_{n} n^{2}\left|s\left(n T-\tau_{2}\right)\right|^{2},} \\
{\left[\mathbf{J}_{e m}\right]_{12}=\left[\mathbf{J}_{e m}\right]_{21}=2 \operatorname{Re}\left\{\frac{1}{\sigma_{1}^{2}} \sum_{n}-j n T s^{*}\left(n T-\tau_{1}\right) s^{\prime}\left(n T-\tau_{1}\right)+\frac{1}{\sigma_{2}^{2}} \sum_{n}-j n T s^{*}\left(n T-\tau_{2}\right) s^{\prime}\left(n T-\tau_{2}\right)\right\},}
\end{gathered}
$$

Note that in the $\left[\mathbf{J}_{e m}\right]_{22}$ element for the FDOA the signal samples are quadratically weighted by time. A dual result for the $\left[\mathbf{J}_{e m}\right]_{11}$ element for the TDOA can be developed where the signal's DFT values are quadratically weighted by frequency; this can be obtained from (21) by using Parseval's theorem and properties of Fourier transforms or can be obtained by using a frequency domain formulation of the signal data as is done in [18]. These quadratically weighted relationships are similar to such weightings shown in the CRB results for the continuous-time case stated in [7] without proof.

\section{References}

[1] W. R. Hann and S. A. Tretter, "Optimum processing for delay-vector estimation in passive signal arrays,” IEEE Trans. Information Theory, vol. IT-19, pp. 608 - 614, Sept. 1973.

[2] W. R. Hann, “Optimum signal processing for passive sonar range and bearing estimation,” J. Acoust. Soc. Am., Vol. 58 pp. 201 - 207, July 1975.

[3] C. H. Knapp and G. C. Carter, "The generalized correlation method for estimation of time delay," IEEE Trans. Acoust., Speech, and Signal Processing, vol. ASSP-24, pp. 320 - 327, Aug. 1976.

[4] P. M. Schultheis and E. Weinstein, "Estimation of differential Doppler shifts,” J. Acoust. Soc. Am., Vol. 66 pp. 1412 - 1419, Nov. 1979.

[5] G. C. Carter, “Time delay estimation for passive sonar signal processing,” IEEE Trans. Acoust., Speech, and Signal Processing, vol. ASSP-29, pp. 463 - 470, June 1981. 
[6] A. H. Quazi, "An overview on the time delay estimate in active and passive systems for target localization,” IEEE Trans. Acoust., Speech, and Signal Processing, vol. ASSP-29, pp. 527 - 533, June 1981.

[7] S. Stein, "Algorithms for ambiguity function processing," IEEE Trans. Acoust., Speech, and Signal Processing, vol. ASSP-29, pp. 588 - 599, June 1981.

[8] E. Weinstein, "Decentralization of the Gaussian maximum likelihood estimator and its application to passive array processing,” IEEE Trans. Acoust., Speech, and Signal Processing, vol. ASSP-29, pp. 945 951, Oct. 1981.

[9] P. C. Chestnut, "Emitter location accuracy using TDOA and differential doppler ," IEEE Trans. Aero. and Electronic Systems, vol. AES-18, pp. 214-218, March 1982.

[10] M. Wax, “The joint estimation of differential delay, Doppler, and phase," IEEE Trans. Information Theory, vol. IT-28, pp. 817 - 820, Sept. 1982.

[11] D. J. Torrieri, "Statistical theory of passive location system,” IEEE Trans. on Aerosp. Elect. Syst., vol. AES-20, no. 2, March 1984, pp. 183 - 198.

[12] B. Friedlander, “On the Cramer-Rao bound for time delay and Doppler estimation,” IEEE Trans. Information Theory, vol. IT-30, pp. 575 - 580, May 1984.

[13] T. Berger and R. Blahut, "Coherent estimation of differential delay and differential Doppler," Proc. ofConf. on Information Sciences and Systems, Princeton University, pp. 537 - 541,1984.

[14] S. Stein, "Differential delay/Doppler ML estimation with unknown signals," IEEE Trans. Signal Processing, vol. 41, pp. 2717-2719, August 1993.

[15] S. Kay, Fundamentals of Statistical Signal Processing: Estimation Theory, Prentice Hall, 1993.

[16] M. L. Fowler and M. Chen, "Evaluating Fisher Information From Data for Task-Driven Data Compression,” Proceedings of Conference on Information Sciences and Systems, Princeton University, March 2224, 2006, pp. $976-972$.

[17] Xi Hu, Mo Chen, and M. L. Fowler, "Exploiting Data Compression Methods for Network-Level Management of Multi-Sensor Systems,” Proceedings of SPIE - The International Society for Optical Engineering, v 6315, Mathematics of Data/Image Pattern Recognition, Compression, and Encryption with Applications IX, 2006.

[18] M. L. Fowler and M. Chen, "Fisher-information-based data compression for estimation using two sensors,” IEEE Transactions on Aerospace and Electronic Systems, vol. 41, no. 3, July 2005, pp. 1131 1137. 